\title{
Reflection and the Path Ahead
}

As the Marshall Journal of Medicine celebrates its first year of publication, there is much to be proud of and even more to look forward to. Before looking ahead, however, I am grateful and would like to offer a word of thanks to our contributors, reviewers, and our Editorial Board for their support of MJM and its mission: to improve the quality of all aspects of comprehensive health care practice of Appalachian rural health medicine.

Launching a new journal is not a small task; it requires an effective team with a common goal and shared vision. I am fortunate to be supported by a team that encompasses these qualities. We achieved a significant measure of success with five on-time issues and consistent, high-quality content from outstanding contributors. Our manuscript turnaround time has averaged 34 days, and we continue to be selective, publishing $62.5 \%$ of our submissions.

The journal has published an average of 10 articles of diverse topics per issue from authors in academic health centers inside and outside of West Virginia. The journal readership map indicates its global readership includes 102 countries with greater than 5,000 downloads since its inception. MJM is currently indexed in Google Scholar, can be easily accessed through CrossRef(USA) and is notably included in BrowZine.

I am pleased to see the steady increase of manuscripts received for publication. This indicates a growing interest among physicians in training, medical educators, researchers, and clinicians. Peer review remains a vital component of our assessment of submitted articles. Due in large part to this dedication, and the high quality of the peer review process, we are optimistic to submit a successful application to the National Library of Medicine for indexing in PubMed after publishing eight issues of MJM.

MJM is a promising model that leads the charge for entities who acknowledge the critical role of the academic institution and its faculty as stewards of academic literature and advocate for equitable access to scholarly research. As the founding Editor-in-Chief, I am delighted to be part of the journal and excited about MJM's opportunity to influence the future through the dissemination of the best ideas and scholarly work of ours educators, researchers, clinicians, and administrators. By providing an academic voice and a venue for discourse, we will move forward in helping to build a world-class scholarly community. 